\title{
Vascular patterns in pterygium and conjunctival autografting: a pilot study using indocyanine green anterior segment angiography
}

\author{
Cordelia M L Chan, Paul T K Chew, Zainah Alsagoff, Jun Shyan Wong, Donald T H Tan
}

Singapore National

Eye Centre

C M L Chan

Department of

Ophthalmology,

National University of

Singapore

P T K Chew

Department of

Ophthalmology,

National University

Hospital, Singapore

Z Alsagoff

J S Wong

Department of Ophthalmology, National University of

Singapore, Cornea

Service, Singapore

National Eye Centre,

and Singapore Eye

Research Institute

D T H Tan

Correspondence to:

Associate professor Donald

T H Tan, Singapore National

Eye Centre, 11 Third

Hospital Avenue, Singapore

168751

snecdt@pacific.net.sg

Accepted for publication

13 September 2000

\begin{abstract}
Aims-To characterise the vasculature of pterygium using indocyanine green (ICG) anterior segment angiography and to demonstrate the pattern of revascularisation following conjunctival autografting. Methods-ICG anterior segment angiography was performed on nine patients with pterygium. Angiography was repeated at 1-2 weeks and 2 months following conjunctival autografting in these patients.

Results-Angiography showed a single feeder vessel originating from the anterior conjunctival circulation in six cases $(66.7 \%)$. This vessel branched to form the radial vessels of the pterygium. Following conjunctival autografting, reperfusion of the vessels in the conjunctival autograft was demonstrable as early as 1 week postoperatively from the episcleral bed. At 2 months postoperatively, the graft appeared well perfused with mild leakage demonstrable at the edges of the graft.

Conclusions-A single feeder vessel from the anterior conjunctival circulation branches to form the radial vessels in pterygium. Reperfusion of conjunctival autografts occurs as early as 1 week postoperatively from the episcleral bed. (Br F Ophthalmol 2001;85:350-353)
\end{abstract}

The use of fluorescein angiography in the evaluation of the anterior segment of the eye has been limited by the problem of leakage even from normal blood vessels, obscuring the arteriovenous and venous phases of the angiogram, thus making interpretation difficult. Meyer and Watson introduced the concept of low dose fluorescein angiography in 1987, overcoming the problem of leakage to a certain extent, but not eliminating the problem entirely, as extravasation is still seen from normal blood vessels in the later phases of the angiogram..$^{1-3}$

The use of indocyanine green (ICG) in ophthalmology has so far been limited to angiograms of the posterior segment. ${ }^{45}$ ICG is a larger molecule than fluorescein (MW $775 \mathrm{Da}$ ) and is more highly protein bound. ${ }^{6}$ These properties would make ICG ideal for use in anterior segment angiography, as negligible extravasation through normal conjunctival and episcleral blood vessels would be expected.

Pterygium is an ocular surface disorder characterised by fibrovascular invasion of the cornea, and a significant propensity towards recurrence after surgical excision. The vascularity of pterygium tissue may have significance in terms of pterygium severity and progression. Tan et al showed that fleshiness of the body of the pterygium, denoted by obscuration of underlying episcleral vessels by the fibrovascular pterygium tissue, was a risk factor for recurrence rate after bare sclera excision, ${ }^{7}$ while the morphology of pterygium recurrence inevitably reflects a high degree of vascularity. As such, we decided to evaluate the blood supply and flow within the fibrovascular component of pterygium, and to determine whether the vascularity is derived from conjunctival or episcleral circulation.

Conjunctival autografting as a surgical option for the management of pterygium has been found to be generally safe and effective. ${ }^{8-10}$ By evaluating the revascularisation and reperfusion of the vessels in the graft, a better understanding of the disease process and factors involved in pterygium recurrence could be developed.

We therefore designed a pilot study and performed ICG anterior segment angiography on patients with pterygium and repeated the angiography following conjunctival autografting in these patients.

Materials and methods

ICG anterior segment angiography was performed on nine patients with primary pterygium, after obtaining full informed consent. The conjunctival and episcleral arterial flow patterns, collateral and venous drainage were characterised. Angiography was repeated at 1-2 weeks and then at 2 months following conjunctival autografting. Angiography was also performed on 1 patient with a normal nasal bulbar conjunctiva as a control.

ANGIOGRAPHIC PROCEDURE

Rapid intravenous injection of $7.5 \mathrm{ml}$ of 50 $\mathrm{mg} / 10 \mathrm{ml}$ ICG Pulsion solution was administered through a butterfly cannula on the 
dorsum of the hand. The angiographic images were acquired using a Topcon 50IA camera with a built in $830 \mathrm{~nm}$ barrier filter, at 35 degree field coverage and maximum gain. The images were digitally processed on an IMAGENet-H1024 digital imaging system. Angiographic images were taken immediately after injection of ICG until the first appearance of the dye was observed. Thereafter, photographs at 2 second intervals were captured for the first 2 minutes, then at 3, 5, and 10 minutes.

TECHNIQUE OF CONJUNCTIVAL AUTOGRAFTING Excision of the pterygium to bare sclera was performed. A free graft obtained from the superior bulbar conjunctiva was placed over the scleral defect and sutured with $8 / 0$ virgin silk. A subconjunctival injection of dexamethasone and gentamicin was given at the end of the procedure. Postoperatively, the patients were prescribed a tobramycin-dexamethasone combination eye drop.

\section{Results}

NORMAL ANGIOGRAM OF THE NASAL BULBAR CONJUNCTIVA

The episcleral vessels appeared early, at 16-25 seconds following intravenous injection of ICG. These vessels were easily identified by
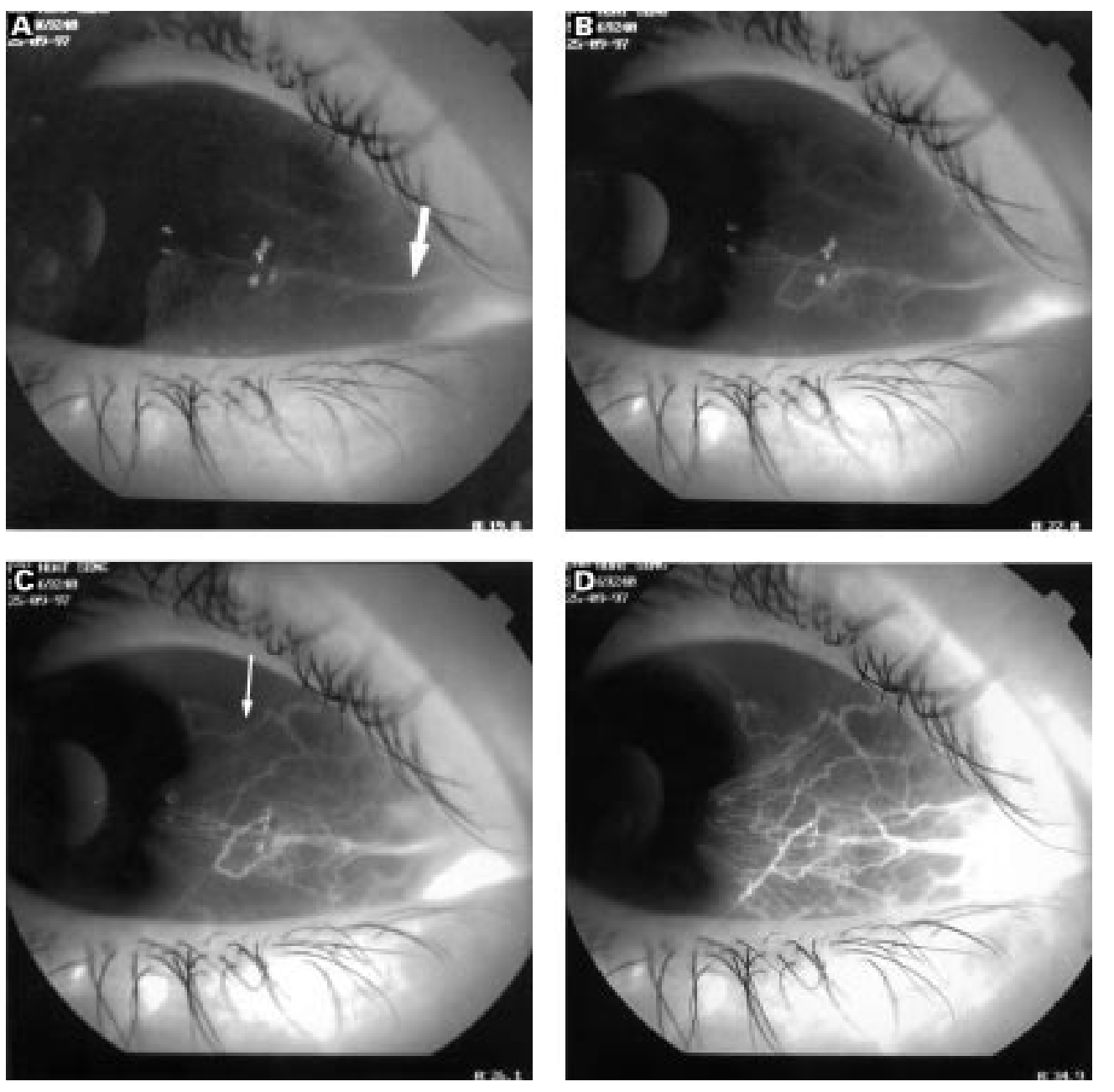

Figure 1 (A-D) Angiographic features in pterygium. The single feeder vessel (thick arrow) branches to form the radial vessels of the pterygium. The episcleral vessels (thin arrow) appear later.

their meandering and tortuous appearance and by correlation with the clinical picture. Vessels from the anterior conjunctival circulation appeared slightly later. These were superficial to the episcleral vessels and appeared straighter. No leakage of dye was observed even beyond 10 minutes of the angiogram.

ANGIOGRAPHIC FEATURES IN PTERYGIUM

A single feeder vessel was found in the early phase of the angiograms in six patients $(66.7 \%)$. This vessel was superficial and straight, indicating its origin from the anterior conjunctival circulation. It appeared between 17.2 and 26.0 seconds after injection of ICG. It subsequently branched to form the radial vessels of the pterygium. The underlying episcleral vessels appeared later, and were highly tortuous and meandering. These appeared between 19.4 and 31.8 seconds following intravenous injection (Fig 1 (A-D)). There appeared to be no communication between the episcleral vessels and the radial vessels of the pterygium from the anterior conjunctival circulation. No leakage of dye was demonstrable from these vessels even in the late phases of the angiogram in all cases. In three cases $(33.3 \%)$ avascular zones were demonstrable at the head of the pterygium.

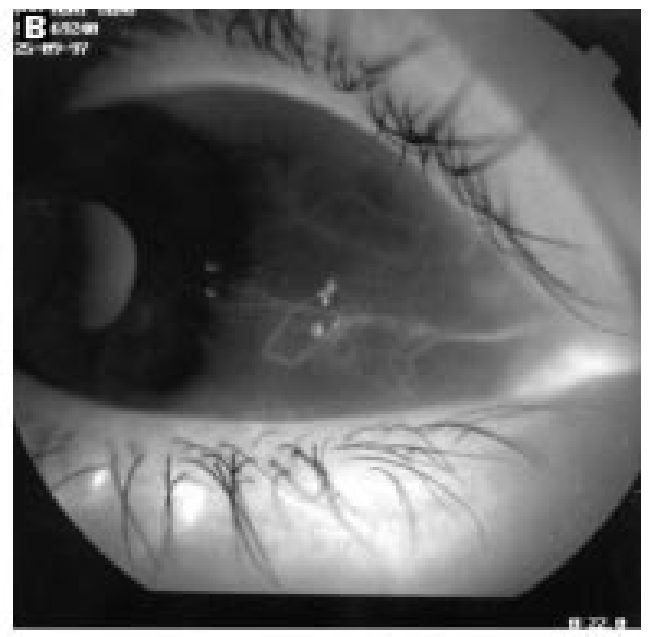



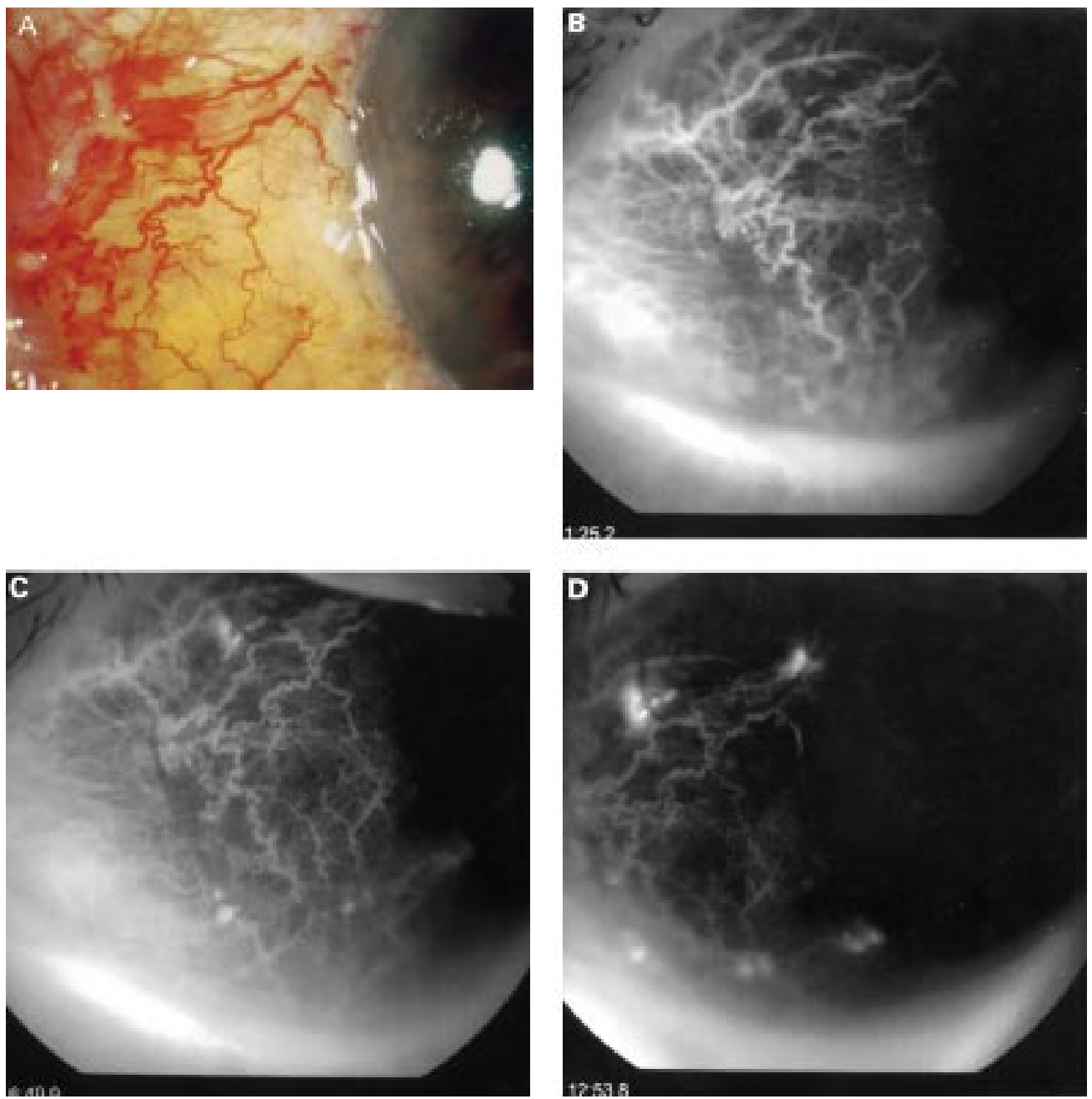

Figure 2 (A-D) Angiogram 1 week after conjunctival grafting. The graft is fully perfused despite obvious gaps between the graft and host conjunctiva in the clinical picture. Leakage of dye is seen at the edges of the graft.

ANGIOGRAPHIC FINDINGS FOLLOWING

CONJUNCTIVAL AUTOGRAFTING

Following conjunctival autografting, angiography was performed at 2 weeks postoperatively in the first seven patients of our study. In all cases $(100 \%)$, the graft vessels were fully reperfused. Perfusion of the graft vessels was noted to commence between 27.5 seconds to 47.5 seconds following injection of the ICG. Gross leakage of dye could be demonstrated at the edge of the graft in six cases $(85.7 \%)$, beginning at between 1 minute 56.2 seconds and 7 minutes 58.9 seconds following injection of the dye. Leakage was not well demonstrated in the seventh case due to the poor quality of the angiogram. Granuloma formation occurred in two of the seven cases and leakage from the granuloma was noted beginning at 3 minutes 38.0 seconds in the first and 5 minutes 49.8 seconds in the second case. The granulomas resolved with topical steroid treatment after 6 weeks.

As a result of our findings at 2 weeks postoperatively, a decision was made to perform the angiogram at 1 week postoperatively in our two remaining cases. Even at 1 week postoperatively, the graft appeared to be perfused, despite obvious gaps between the graft and the host conjunctiva in the clinical picture (Fig 2
(A-D)). This would imply early reperfusion from the episcleral bed beneath the graft. Reperfusion commenced at 23.2 seconds in the first case and 35.0 seconds in the second case.

Angiography was again repeated at 2 months postoperatively. This consistently showed a well perfused graft with mild leakage from the edges.

\section{Discussion}

ICG is a water soluble tricarbocyanine dye primarily bound to globulins ${ }^{611-13}$ As it is a larger molecule and more highly protein bound than fluorescein, it is not as readily extravasated from the choriocapillaris, making ICG angiography useful in the evaluation of chorioretinal disorders. $^{45}$

A pilot study by Alsagoff et al (in preparation) successfully used ICG in anterior segment angiography to characterise conjunctival and trabeculectomy bleb vascular status, demonstrating its potential usefulness as a research or investigational tool. In our study, clear, well defined, high contrast angiograms of the conjunctival and episcleral circulation of the nasal bulbar conjunctiva were obtained. No leakage from the episcleral and conjunctival vessels was seen throughout the early and late 
stages of the angiogram in our normal control. This made it possible for us to evaluate the filling patterns in pterygium.

Morphological studies on pterygium thus far include histological evaluation with the electron microscope, evaluating the epithelial ultrastructure, and morphological observation of the intraepithelial capillaries in pterygium. ${ }^{14}$ However, these have yet to define the blood supply and filling patterns in pterygium and it is still unclear as to whether pterygium is a disease of the conjunctival or episcleral circulation.

In our study, a single feeder vessel was seen in $66.7 \%$ of our cases. This vessel was superficial and straight and, by correlation with the clinical picture, appeared to arise from the conjunctival circulation. This would imply that pterygium derives its blood supply from the anterior conjunctival circulation and not from the episcleral circulation. However, our study is limited by its small sample size, especially when the vascular patterns in pterygium are highly variable. If significant, therapeutic ablation of this feeder vessel could be a promising treatment modality of the future.

The early reperfusion of the conjunctival autograft at 1 week postoperatively despite clinical evidence of gaps between the graft edge and host conjunctiva would mean early reperfusion of the graft from vessels of the underlying episcleral bed. Excessive cautery of the episcleral bed following excision of the pterygium should therefore be avoided, as this would delay reperfusion of the graft, and could explain the prolonged graft oedema seen in some cases postoperatively.

In our small series of nine cases, no recurrence of the pterygium occurred during our study period and so the filling patterns of recurrence could not be evaluated.

\section{Conclusions}

The blood supply of pterygium is derived from the anterior conjunctival circulation, as evidenced by the single feeder vessel appearing early in the angiograms in our study. This vessel branches to form the radial vessels seen in pterygium. More studies with a larger sample size would be required to further elucidate the nature and variable vascular patterns in pterygium. Following conjunctival autografting, the graft is perfused as early as 1 week postoperatively, from the underlying episcleral bed.

This study was performed in the Singapore National Eye Centre and the Department of Ophthalmology, National University Hospital. It was supported by a grant from Singapore Eye Research Institute.

1 Brunn-Jensen N. Fluorescein angiography of the anterior segment. Am f Ophthalmol 1969;67:542-5.

2 Meyer PAR, Watson PG. Low dose fluorescein angiography of the conjunctiva and episclera. Br F Ophthalmol 1987;71: $2-10$.

3 Meyer PAR. Patterns of blood flow in episcleral vessels studied by low-dose fluorescein videoangiography. Eye 1988;2:533-46.

4 Guyer DR, Puliafito CA, Mones JM, et al. Digital indocyanine-green angiography in chorioretinal disorders. Ophthalmology 1992;99:287-91.

5 Destro M, Puliafito C. Indocyanine green videoangiography of choroidal neovascularization. Ophthalmology 1989;96: $846-53$.

6 Fox IJ, Wood EH. Indocyanine green: physical and physiologic properties. Proc Mayo Clin 1960;35:732-44.

7 Tan DTH, Chee SP, Dear KBG, et al. Effect of pterygium morphology on pterygium recurrence in a controlled trial comparing conjunctival autografting with bare sclera excision. Arch Ophthalmol 1997;115:1234-40.

8 Kenyon KR, Wagoner MD, Hettinger ME. Conjunctival autograft transplantation for advanced and recurrent pterygium. Ophthalmology 1985;92:1461-70.

9 Chen PP, Ariyasu RG, Kaza V, et al. A randomized trial comparing mitomycin $\mathrm{C}$ and conjunctival autograft after excision of primary pterygium. Am f Ophthalmol 1995;120: $151-60$.

10 Starck T, Kenyon KR, Serrano F. Conjunctival autograft for primary and recurrent pterygia: surgical tehnique and problem management. Cornea 1991;10:196-202.

11 Baker KJ. Binding of sulfobromophthalein (BS) sodium and indocyanine green (ICG) by plasma alpha-1 lipoproteins. Proc Soc Exp Biol Med 1966;122:957-63.

12 Benson RC, Kues HA. Fluorescence properties of indocyanine green as related to angiography. Phys Med Biol 1978;23:159-63.

13 Kogure K, David NJ, Yamanouchi U, et al. Infrared absorption angiography of the fundus circulation. Arch Ophthalmol 1970;83:209-14

14 Seifert P, Sekundo W. Capillaries in the epithelium of pterygium. Br f Ophthalmol 1998;82:77-81. 